\title{
The Role of Wind Forecasting in Grid Operations \& Reliability
}

\author{
Mark L. Ahlstrom, Member, IEEE, and Robert M. Zavadil, Member, IEEE
}

\begin{abstract}
Wind energy is increasingly viewed as a desirable energy source, but it must be integrated into power grids and electric utility systems that are understandably oriented around issues of power, capacity and reliability. It is important to know the actual impact costs of significant wind penetration levels, and the paper first describes a study approach and the results of a specific wind integration study in North America. Once the impact costs are understood, it is then possible to design tools and operating practices to minimize these impact costs. Wind forecasting tools are a critical element in minimizing the impact costs and ensuring reliability with larger amounts of wind energy on the grid, and the approaches and reasonable expectations for state-of-the-art wind forecasting methods are described. The value of wind energy is increased, and larger amounts of wind energy can be used, if it can be scheduled using wind forecasting methods.
\end{abstract}

Index Terms - Costs, Forecasting, Power generation dispatch, Power system reliability, Wind, Wind energy, Wind power generation

\section{INTRODUCTION}

A significant obstacle to the development of wind generation stems from the fact that utility systems are necessarily biased toward resource capacity - the rate of energy transfer to the grid, not just the total amount delivered over a longer period of time - to insure the adequacy, reliability, and security of the electric supply.

Integrating large amounts of wind energy into the larger portfolio of electric generation resources requires some special considerations on the part of those charged with operating the electric system. Substantial amounts of wind generation in a utility system can increase the demand for various actions and services. The ability of the control area to provide the required level of these services for successful integration, and their additional cost of doing so, depends on the makeup of its generating fleet, agreements with neighboring control areas, or the existence of competitive markets for such services.

Understanding and quantifying the impact of wind energy on specific electrical systems is a critical issue. The term

M. L. Ahlstrom is with WindLogics Inc., 1217 Bandana Boulevard North, Saint Paul, MN 55108 USA (e-mail: mark@windlogics.com).

R. M. Zavadil is with EnerNex Corporation, 144-E Market Place Boulevard, Knoxville, TN 37922 USA (e-mail: bobz@enernex.com). "impact" is generic, but generally includes any additional costs borne by the operators of the power system resulting from the variability and "predictability" of wind generation. While wind energy may not be "dispatchable", the cost impacts of wind can be substantially reduced if the wind energy can be scheduled using wind forecasting.

There are very good reasons for using more wind energy on electricity systems, but we must understand how the wind energy interacts with the systems, power markets and operators. Once we understand the impacts, we can then design the rules, tools and forecasting systems to minimize cost impacts and maximize the value of wind energy on the system.

\section{WIND FORECASTING IN GRID OPERATIONS \& RELIABILITY}

\section{A. Background on Utility Impacts of Wind}

The nature of its "fuel" supply distinguishes wind generation from more traditional means for producing electric energy. The electric power output of a wind turbine depends on the speed of the wind passing over its blades. This moving air stream exhibits variability on a wide range of time scales from seconds to hours, days, and seasons. Local and regional weather patterns, seasonal variations, terrain and other nearby turbines are just a few of the factors that can influence the electrical output variability of a wind turbine generator.

From the perspective of the power system, the aggregate performance of a large number of turbines rather than an individual turbine is generally more important. Wind turbines are usually spread out over a significant geographical area within the wind plant, and multiple wind plants are distributed over a much larger area within the control area. This spatial diversity has the beneficial effect of smoothing some variations in electrical output.

Another aspect of wind generation, which applies to conventional generation but to a much smaller degree, is the ability to predict with reasonable confidence what the output level will be at some time in the future. Conventional plants, for example, cannot be counted on with $100 \%$ confidence since mechanical failures or other circumstances may limit their output. The probability that this will occur, however, is low enough that such an occurrence is often ignored in shortterm planning activities.

Because wind generation is driven by the same physical phenomena that control the weather, the uncertainty associated with a prediction of future generation is more 
significant. The combination of production variability and higher uncertainty of prediction can make it more difficult to fit wind generation into established procedures for power system operations, planning and scheduling.

On most large power systems, multiple individual "control areas" coordinate their activities to maintain reliability and conduct transactions of electric energy with each other. A control area consists of generators, loads, and transmission ties to neighboring areas. Each control area must assist the larger interconnection with maintaining frequency and balance load, generation, out-of-area purchases and sales on a nearly continuous basis. In addition, a prescribed amount of reserve capacity must be maintained at all times as protection against unplanned failure or outage of equipment. To minimize costs while ensuring system performance and reliability, each control area is continuously developing plans and schedules for meeting the forecast load while honoring all technical constraints and contractual obligations. The operators of the control area monitor the operation of the control area in real time and make adjustments when the actual conditions deviate from those that were forecast. These adjustments and deviations from the "optimal" plans and schedules may add incremental operating costs.

Operating the power system involves more than adjusting the combined output of the supply resources to meet the load. Maintaining reliability and acceptable performance requires that operators:

- Keep the voltage at each node of the system within prescribed limits;

- Regulate the frequency (the steady electrical speed at which all generators in the system are rotating) of the system to keep all generating units in synchronism;

- Maintain a state that can withstand and recover from unplanned failures of major elements to prevent cascading outages or forced blackouts.

The control area operator actions and functions necessary for maintaining system performance and reliability are referred to as "ancillary services". These services cost money to provide, and there may be no direct mechanism for recovering these costs.

Strategies for operating the power system are based on forecasts of conditions to come, whether it is seasons, days, or minutes ahead. Uncertainties abound in the input data upon which these strategies are developed, and uncertainty regarding the aggregate load to be served at each instant over the planning horizon is a major factor. Sophisticated algorithms are used to forecast the load, and an optimal plan is created for meeting that load. If the actual load deviates significantly from the forecast used to develop the plan, there is a high likelihood that the cost to serve that load will be higher than with an optimized plan.

The question for wind generation is how its variability and the accuracy with which it can be forecast affect the deployment and operation of other generating resources in a control area, and how the cost of those operational modifications affects the overall cost to serve the load. One does not have to look too far back to recall claims that wind plants need " $100 \%$ backup, because they are not reliable." While the wind and power industries have, for the most part, moved beyond such claims, it is difficult to argue that the presence of wind generation in a control area would not require some prudent adjustments to operating strategy. The objective now has really turned to data and methods for quantifying the economic impacts of these changes, along with assessing what technical impacts wind generation may have on system performance and reliability, and engineering solutions to minimize these impacts.

\section{B. Cost Impact Example - The Xcel Wind Integration Study}

Xcel Energy is the fourth-largest combination electricity and natural gas energy company in the United States. Its northern control area, the former Northern States Power (NSP) system, serves over 1.4 million electric customers in the states of Minnesota, Wisconsin, North Dakota, South Dakota and Michigan. Peak demand in the control area was approximately $9,000 \mathrm{MW}$ in 2003 and is projected to rise to approximately 10,000 MW by 2010 .

This operating area currently has about $300 \mathrm{MW}$ of wind operating in Southwestern Minnesota. An earlier study commissioned by Xcel Energy and the Utility Wind Interest Group (UWIG, www.uwig.org) estimated that this $300 \mathrm{MW}$ of wind generation results in additional annual ancillary services costs to Xcel of $\$ 1.85$ for each megawatt-hour (MWH) of wind energy delivered to the system.

An additional $170 \mathrm{MW}$ of wind power is under contract and $450 \mathrm{MW}$ had been awarded through a 2001 All Source Bid process. Partially as a result of Minnesota legislation, it is expected that this system will have $1500 \mathrm{MW}$ of wind power by 2010 and as much as 2,250 MW by 2015 . This could represent one of the highest wind penetrations of any North American power system.

A new study was commissioned by the Minnesota Department of Commerce and Xcel Energy in 2004 to assess how larger amounts of wind generation would affect Xcel operating costs in the NSP control area. Unlike the first study, the scenario included wind plants not yet in operation and dispersed over a considerable geographic region. Determining how the aggregate wind generation would appear to the Xcel control area operators was a critical aspect of the analysis.

To create a detailed and realistic chronological representation of the projected wind generation facilities for the study, sophisticated meteorological simulations were used to "recreate" the weather for recent historical years. This was done using large archives of the hour-by-hour historical weather data to initialize and continuously adjust a numerical simulation model of the atmosphere in the region of interest. While the base model is very similar to those used by various agencies for short-term weather forecasting, special techniques allow the spatial resolution of the model to be increased in and around the locations of the prospective wind generation facilities. 
On the innermost grid, specific points that were either colocated with existing wind plants or likely prospects for future development were identified. Wind speed data along with other key atmospheric variables from these selected locations (Figure 1) were saved at ten-minute intervals as the simulation progressed through three years of weather.

The high-resolution time series of wind speed data was converted to wind generation data by applying power curves for existing and prospective commercial wind turbines. As a check on the accuracy of this approach, the calculated wind generation data was compared to actual measurements from groups of turbines at an existing wind plant in the area for the entire year of 2003 to validate the models.

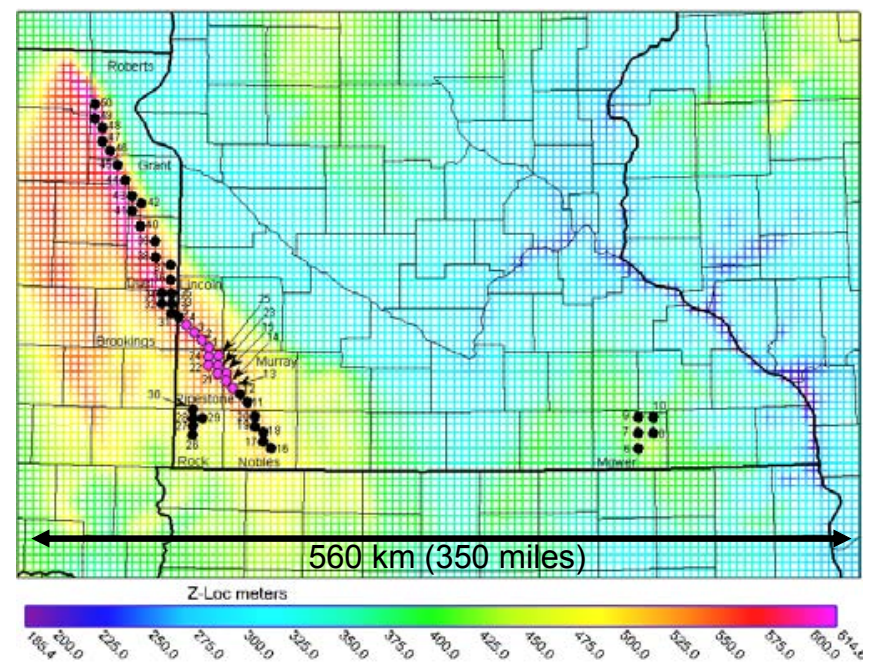

Fig. 1. This image shows the wind plant locations (in the USA states of Minnesota and South Dakota, mostly on the area known as the Buffalo Ridge) The detailed weather and wind plant energy production was derived from years of hourly data in 3-dimensional gridded weather archives. Using physics-based weather modeling methods and racks of computers running for many weeks, three years of hub-height wind data was generated at 10 -minute time steps.

Because this approach is directly based on real weather data, it does an outstanding job of representing key items that are critical to the analysis:

- Wind plant energy is properly "time synchronized" with other utility operating data, which is important since correlations exist between weather events and system data (including load).

- This physics-based modeling approach captures the effects of geographic dispersion of wind plants and other relationships between the individual plants.

Three years of day-ahead wind forecasting data for all fifty wind plant locations were also developed from the model. This was used to study unit commitment issues and costs for wind energy using a wind forecast. Additional forecast experiments showed the value of more sophisticated forecasting methods using computational learning systems with numerical weather models to significantly improving the accuracy of forecasts spanning a range from hours to days ahead. With subsequent funding, we are now working on the development and demonstration of these advanced systemwide forecasting methods.

\section{Xcel Study Results}

The high-fidelity data from the meteorological simulations provided a very solid basis for various analysis of how the operation of the Xcel control area in Minnesota would be affected by the proposed wind generation. Specific impacts that were analyzed included:

- How much additional regulation capability would be required due to the addition of wind generation? Regulation is generating capacity allocated to follow the fastest fluctuations in control area demand. Units on regulation respond to automatic commands over tens of seconds to a few minutes.

- How does fluctuating wind generation affect the amount of controllable generation required to follow slower changes - tens of minutes to hours - of the control area demand as it proceeds through a daily pattern?

- How does the uncertainty associated with wind generation forecasts affect the commitment and scheduling of generation for the next day or days, and what are the economic consequences?

Wind generation data from the meteorological simulation model matched with historic load and generation data from Xcel's archives provided for very straightforward analytical approaches to the major study questions. Control area regulation requirements were assessed by analyzing the statistics of the fast fluctuations in existing load and comparing them to similar fluctuations in high-resolution measurement data from wind generation facilities in Minnesota. Since fast changes in wind and load are uncorrelated, the regulation requirement of the projected control area load combined with wind generation can be calculated with some simple algebra. The addition of 1500 MW of wind generation to the Xcel control area increases the system regulation requirement by only $8 \mathrm{MW}$, from 60 to 68 MW.

Because the synthesized wind generation and historical load data were time synchronized, the effect of wind generation on longer-term (tens of minutes to hours) changes in control area demand was analyzed by comparing the changes in the load alone to those in the load combined with wind generation. It was found that wind generation has only a small effect. Further mathematical analysis of these distributions reveals that only a small increase in generation ramping capability would be necessary to prevent degradation of control performance.

Finally, because most electrical generating systems cannot be stopped or started at will, control area operators must develop an optimized plan for the coming days. This plan must have the lowest cost while still honoring the constraints of individual generating units (permissible stops/starts, ramp 
rates, etc.) and the control area itself (e.g. spinning reserve, operating reserve, etc.). While system operators are quite familiar with the patterns of load in their control area, wind generation introduces some significant unknowns into this process. Using the synthesized wind generation and historical load data, the effect of wind generation on the hour-by-hour control area demand pattern can be assessed, with some surprising results.

To quantify the cost impact, we mimicked the activities of the system schedulers, then calculating the costs of the resulting plans. The input data for the analysis was hourly load data, wind generation data, and wind generation forecast data for a two year period. For each day, a reference case was developed that assumed that the daily energy from wind generation was known precisely, and that it was delivered in equal amounts over the 24 hours of the day. This conservative reference case was selected since it represents wind as a resource that would have the minimum impact on the operation of other resources

Next, we represented the actions of the system schedulers. The projected load and hour-by-hour wind generation forecast were input to the unit commitment and scheduling program. The program determined the lowest cost way to meet the load and accommodate the wind generation as it was forecast to be delivered. The forecast wind generation was then replaced by "actual" wind generation and a simulation of the same day was conducted. However, instead of allowing the program to change the planned deployment of generating resources, only the resources available in the original plan could be used to meet the actual load.

Applying this method to over 700 days of wind generation and system load data, it was determined that Xcel Energy was effectively paying an additional US $\$ 4.37$ for each MWH of wind energy. These costs were incurred in the form of additional production costs to Xcel (cost to serve the load not served by wind generation).

These results assumed the conservative use of current operating practices and a "modest effort" wind energy forecast. It is very likely that these costs would be reduced significantly through modifications to operating practices, use of energy markets and improved forecasting methods.

\section{Value of Wind Forecasting}

Wind forecasting is increasingly showing value for the improved scheduling of wind energy, and as shown above, such forecasts can have substantial value even if they are not perfectly accurate. Using physics-based forecasting models, real-time wind and energy data from the wind plants, and computational learning systems such as artificial neural nets or support vector machines, it is possible to provide forecasts of wind energy delivery that are significantly better than simplistic forecasts based on climatology (historic values) or persistence (assuming that what is currently happening will continue without change).

The accuracy of forecast results depends on the specific challenges of the wind plant location, the surrounding terrain and the local climatology. Because wind plants are intentionally located at sites that tend to amplify wind effects when compared with the surrounding space, it is often worthwhile to run customized fine-scale forecasting models and local wind flow models to simulate the enhanced local effects. These results may be combined with other regional forecasts using computational learning systems to detect complex relationships and optimize the wind energy forecast.

Typically, the operator will want to receive forecasts that run hour-by-hour for the next few days to reduce the unit commitment costs that were described above. While results are site specific, a reasonable expectation at most locations is that the accuracy of "next day" hour-by-hour power forecasts using current state-of-the-art methods will have a mean absolute error (MAE) of perhaps $10-15 \%$ of the rated (nameplate) capacity of the wind plant.

For the purposes of supporting reliability and providing information to real-time operators of the grid, it is also important to provide forecasts of the coming hours with high temporal resolution. While the cost impact on many systems may be primarily in the next day timeframe, operators clearly want to know what is expected to happen in the next few hours, especially with regard to more dramatic (but infrequent) events such as rapid shifts in wind energy production from storm events. It is quite possible to update the forecast every hour to provide additional detail for the next few hours at a resolution of every 10 or 15 minutes, and when it provides value, special "rapid update cycle" forecasting methods may also be used that run every hour to provide updated results for the next few hours. Accuracy of power forecasts for the next few hours can typically have mean absolute errors on the order of $5 \%$ of rated wind plant capacity.

It is worth noting that the accuracy of energy forecasts (e.g., total wind energy for tomorrow) can be significantly better than the accuracy of power forecasts. The exact timing of the passage of weather fronts can be difficult to predict, and because the winds can change with the passage of such fronts, errors in timing can result is large power errors. These errors tend to balance out over longer periods of time such as several hours or a full day, so the accuracy of energy forecasting can be quite good. To the extent that wind energy is balanced with gas purchases or hydro generation, this can be used to advantage.

It is also important to note that forecasting errors are significantly reduced when aggregated on a system-wide basis. Due to the smoothing effects of geographic dispersion, system-wind forecasting errors for multiple dispersed wind plants may be reduced by perhaps $30-50 \%$ when compared with the errors of individual wind plants.

\section{E. Summary \& Conclusions}

High-resolution wind speed data from meteorological simulations combined with historical utility system data provides a realistic foundation for assessing wind generation impacts on electric system operations. A similar approach can be extended to studies of transmission and power market 
issues. This is a powerful technique for determining the impact costs and operating impacts of additional wind generation.

The results from the Xcel study provide some interesting insights. For example:

- Cost impacts in regulation and load following timeframes were very modest.

- The variability of wind generation in sub-hourly time scales would have little technical impact on the performance of the control area.

- Cost impacts were largest in the next day planning time frame, and could likely be reduced with adjustments to operating strategy, improvements in wind forecasting and access to real-time power markets.

This study approach is data- and computer-intensive, but there is great value in accurately estimating wind energy impacts from a cost-based perspective. By understanding the impacts, we can work toward maximizing the value of wind energy and providing tools for the operation of the grid and the power system to minimize the cost impacts and the operational concerns.

Using state-of-the-art wind forecasting is one of the key tools to minimize the cost and operating impacts of wind energy and to facilitate increasing penetrations of wind energy on power systems. Wind energy is a valuable part of the generation mix and in many situations it may actually be one of the lowest cost sources of energy. Advances in wind forecasting will allow use of increasing amounts of this important energy resource on our power systems while addressing the vital issues of system reliability, capacity and cost.

\section{ACKNOWLEDGMENT}

A broad-based workgroup was assembled to guide the initial development of the Xcel Wind Integration Study, and while they are too numerous to name individually here, we wish to convey our sincerely thanks to this group for their contributions. This group included representatives of Xcel Energy, Minnesota municipal utilities, Minnesota cooperative utilities, the Minnesota Chamber of Commerce, the American Wind Energy Association, Minnesota environmental organizations, the U.S Department of Energy / National Renewable Energy Laboratory, and the Department of Commerce. The project team itself also included many other people, including those from EnerNex (Jack King, Leo Xiadong), WindLogics (Dr. Bruce Lee, Dr. Dennis Moon, Dr. Cathy Finley, Lee Alnes), Arreva T\&D (Dr. Lawrence Jones, Fabrice Hudry, Mark Monstream, Stephen Lai) and NexGen Energy/Utility Wind Interest Group (J. Charles Smith).

\section{REFERENCES}

[1] R. M. Zavadil et al, "Xcel Energy and the Minnesota Department of Commerce - Wind Integration Study - Final Report," EnerNex Corporation and WindLogics Inc., September 28, 2004. Available: http://www.windlogics.com.

[2] B. Lee, D. Moon, R. M. Zavadil et al, "Xcel Energy and the Minnesota Department of Commerce - Wind Integration Study - Task 1 Report Characterization of the Wind Resource in the Upper Midwest," EnerNex Corporation and WindLogics Inc., September 10, 2004. Available: http://www.windlogics.com.

\section{BIOGRAPHIES}

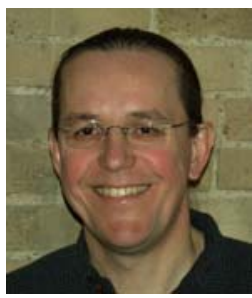

Mark L. Ahlstrom is CEO at WindLogics Inc. (www.windlogics.com) of Saint Paul, Minnesota, USA. Under his leadership, the company has become a leader in applied meteorology, wind energy analysis and wind forecasting. Prior to joining WindLogics, Mark was founder and President of Keller Group, a provider of fax and voice software technology to IBM, Global Village Communications and others. Previously he founded Airplan Systems, the developer of decision support front-end technology for airline reservation systems, which he sold to Continental Airlines. Mark holds degrees in Biochemistry (BS 1979) and Biomedical Engineering (MS 1981) from the University of WisconsinMadison and was a research scientist in the Artificial Intelligence group at the Honeywell Computer Sciences Center. He is a member of the IEEE Power Engineering Society.

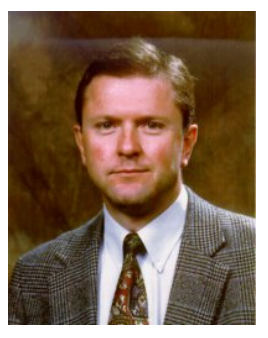

Robert M. Zavadil (M 1982) received a BSEE from South Dakota State University in 1982 . He began his power industry career with the Nebraska Public Power District (NPPD) as a special studies engineer and was engaged in a variety of technical activities involving transient and harmonic simulation, power quality, substation ground, and RFI/TVI from high voltage transmission facilities. He joined Electrotek Concepts, Inc. in 1989. At Electrotek, Mr. Zavadil consulted for a range of clients including the Electric Power Research Institute, the National Renewable Energy Laboratory, major U.S. electric utilities, and private corporations. His technical expertise includes distribution system and end-user power quality and power electronics, motor and drive technology and applications, power quality and power quantity measurements, and the application of computer techniques and simulations for solving power system problems. He co-founded EnerNex Corporation in 2003. He is a member of the IEEE Power Engineering, Power Electronics, and Industrial Applications Societies 